ner $^{17}$ found atrophy of the skin of the left side of the face in an epileptic lady.

After having considered the condition of the nervous system of our patients, it appears that the cause of these disturbances of the skin, have to be found in that system. The action of the nerves is to regulate the functions of the blood vessels. The alterations seem to be especially on the sympathetic which presides over the vegetative functions, but we can not absolutely deny that the nervous centers are implicated in the production of these affections. Fibers from the brain and from the spine go through the ganglia which form the origin of the sympathetic, and make it an independent system. The fibers coming out of the ganglia are not only motor, but they are also of a sensitive nature. This is plailily emphasized when we see that an irritation applied on an organ under the control of the sympathetic causes to increase the movements of the organ supplied with that group of nerves. The ganglia are not organs of movement, but they are organs where the reflex action is accomplished without any knowledge of the brain. Any stimulus applied to the sensitive fibers, causes a reflex movement through the ganglia, the sensation remaining in it without causing any feeling. But when the stimulus exceeds the normal point it becomes an irritation, its effect does not remain limited to the ganglion, but is carried to the spine and to the brain, thus producing sensation. Many disturbances of the nutrition of the skin generally happen without producing sensation, remaining strictly limited in the jurisdiction of the sympathetic. This we see when the disturbance is not very intense, as in our case of leucodermia or liodermia, but when the results of the affection are deeper like in scleroderma, then some sensation is aroused, like in one case which was accompanied with neuralgic pains and itching sensations.

There is no doubt that some of these affections, by their topographic distribution on the skin, reminds the ramifications of the nerves laying underneath, or better, they follow the lines of division of the cutaneous nerves as drawn by Voight. In nevus line ris, ichthyosis cornea, hystrix, we find the disposition of the disease pursue the cause of the underlaying nerves. In one case of ichthyosis hystrix which came under my observation in a boy eight years old, the disease so closely followed the course of the sciatic and crural nerves in these regions of the skin that it looked as if somebody had drawn lines with the pencil. In many cases of anomalies of the pigment as in nevi pigmentarii, either congenital or acquired short time after birth, we see the lines wonderfully follow the ramification of the nerves of the affected region. Kaposi refers to two cases of nævi verrucosi, where the verruca were seated precisely on the course of the hyschiatic nerve.

Neumann ${ }^{18}$ referred to a case of nervous nevus, where the pigment hypertrophy and the warts were distributed from the gluteal region to the toes of the foot in perfect correspondence with the nervous ramifications of the leg. The disposition however, of these maculæ or hypertrophies of the epidermis reminds that of the underlaying nerves. According to Phillipson, ${ }^{19}$ the skin disturbances follow rather the cutaneous nerves maintaining the lines of Voight. The difference of direction between some lines of altered

18 Oesterr, Jahrbuch f. Pædiatrick, 1877.

19 Monaushelfte für Pract. Dermatologie. 1890. skin and the direction of the nerves, would be explained by the physical growth of the body, the affection remaining of the size as it was in infancy.

The nerves which control the nutrition of the skin are not distinct from others, but are in connection with motor and sensory filaments. In consequence of this fact, Baerensprung considered alterations of the skin of this kind the result of a disturbance of a spinal nerve. The same we can say in the nævivasculares, when the spots represent an area which recalls the nerve of the region. Nevus vascularis of one side of the face shows that originally in the embryo the trigeminus was affected and the dilatation of the capillary blood vessels was the result.

In our first case we have two areas of the skin affected with atrophy in an oblong form. It begun with a small spot of a reddish violet color, which gradually got white and atrophic in the center, showing the intensity of the color in the periphery. The intense redness was the result of stasis, due to the diminished tonus of the blood vessels, the vasomotor nerves having lost their control.

In the second case we have atrophy of the pigment following the course of the maxillary nerve inferior of one side and on the neck, breast, arm and hands of both sides.

In the third case we remarked a deep alteration of the skin affecting the corium and the subcutaneous tissue and muscles, due to the condition of nutrition of the limbs and on body.

All the three affections came recently, slowly progressing in persons affected with nervous diseases, and accompanied with nervous symptoms as anæsthesia, analgesia, hyperæsthesia.

I believe that lioderma, leucoderma and scleroderma, although different affections have great similarity between themselves. They are affections which attain people suffering with an altered condition of the general nervous system. They are the result of an affection of the nerves either central or peripheric. In one word they are the result of an alteration of nutrition of the skin by altered conditions of the trophoneurotic nerves. In conclusion I would say that the difference betweeen leucoderma, lioderma and scleroderma is only of degree, produced by lack of nutrition affecting in the first the ameboid cells of the malpighi layer which furnishes the granules of the pigment, in the second affecting the connective tissue of the superficial layer of the corium, and finally in the third involving the connective tissue of the corium and of the subcutaneous tissue.

\section{MEDICINAL PLASTERS.}

BY JOHN V. SHOEMAKER, A.M., M.D.,

PROFESSOR OF MATERICA MEDICA, PHARMACOLOGY, THERAPEUTICS AND CLINICAL MEDICINE, AND CLINICAL PROFESSOR OF DISEASES OF THE SKIN IN THE MEDICO-CHIRURGICAL COLLEGE OF PHILADELPHIA;

In most diseases involving the skin both constitutional and local treatment is needed in order to effect a cure or improvement. Topical remedies may be applied in different forms and it is often a question whether a powder, a solution, an ointment, paste or plaster is calculated to afford most speedy relief. Plasters possess certain advantages which render them appropriate means of dealing with some varieties of skin diseases. They are especially adapted to the treatment of chronic and localized conditions. In 
the subacute and formative stages of some maladies they are also of value.

Plasters are composed of mixtures which are solid at ordinary temperatures but which soften somewhat when kept in contact with the skin, to which they closely adhere. The medicated material is spread evenly upon some fine texture, very fine muslin being usually employed.

Plasters constitute an efficient protection of the diseased area against the atmosphere. Bacilli which float in the air are constantly being deposited upon the general surface, and some of these microörganisms need but a fitting soil in order to become exciting causes of disease. If, therefore, the parts are first thoroughly cleansed by a disinfectant solution the application of a plaster is an aseptic procedure. It may, furthermore, be made an antiseptic dressing by virtue of the materials which it contains. This quality not infrequently enables plasters to arrest the progress of a beginning inflammation. It enables them, moreover, to protect the affected part from a secondary or mixed infection.

A plaster maintains itself constantly in position and, consequently, the active medicaments which it contains have the most favorable opportunity to demonstrate their efficiency. When applied to properly selected cases a plaster very seldom excites irritation or aggravates the malady by the development of too much heat or by confining a discharge. If any fear of such undesirable action should arise the dressing may be easily removed every day or two and the surface cleansed with oil, glycerine, water, soap or alkaline solution. The plaster may then be reapplied. On parts in which the morbid action is sluggish the dressing may safely be left in place for several days or a week. This comparative permanence of fixation is an advantage appreciated by both patient and physician.

Plasters can be so accurately adjusted to any spot that they are for this reason a cleanly dressing. This is a virtue which every one will appreciate who has seen underwear and bed clothing soiled by the use of lotions or ointments. Their cleanliness is an undeniable convenience in precisely those chronic cases to which they are, in general, most appropriate. The fine back of the dressing also guards the surface against friction.

In consequence of the adhesiveness of plasters they can be effectively used upon portions of the body to which powders, lotions and ointments cannot be conveniently applied or maintained in position. This is the case, for instance, with the face, lips and feet. A clean-backed patch of plaster upon the face is certainly a less unsightly application than a smear of ointment. An ointment will likewise in most cases be unpleasant when rubbed upon the lip. Again, plasters are particularly beneficial in the treatment of fissures. They bring the medicament in contact with the fissure and maintain its action continuously as long as needed. They approximate the edges of the crack in such a manner as to promote repair. And as fissures are, in many instances, due to the nature of the patient's occupation, plasters prevent contact of water or other more excitant fluid with the lesion. Plasters place the borders of the tear at rest and prevent them from keeping it open for an indefinite perjod.

These applications exert a gentle compression which in appropriate cases has a beneficial influence upon the capillary circulation of the part. For the same roason they stimulate the absorbent vessels to remove morbid deposits.

Finally, plasters exert an influence upon nerve endings and may, therefore, be utilized for the alleviation of pain.

These preliminary considerations lead me to the enumeration of those combinations which I have with benefit employed. In connection with the description of their composition I will briefly name the conditions in which I have used the various plasters with advantage.

The basis of these preparations, the plaster-mass with which actively medicinal substances are incorporated, consists of 50 parts of india rubber, 5 parts of honey and 45 parts of soap-plaster.

LIST OF MEDICINAL PLASTERs.*

Anthrarobin.-A plaster containing 25 per cent. of anthrarobin in 75 per cent. of plaster mass is employed with success in chronic eczema, psoriasis, acne, rosacea, old syphilitic and scrofulous patches and old ulcers.

Antimony.-Antimony, 10 per cent., plaster mass, 90 per cent. Antimony plaster is a good application in indurated spots of aene and eczema, chloasma, scars and keloid.

Alum, ergot, hemlock and white precipitate.-This is composed of 20 per cent. of alum, 20 per cent. ergot, 20 per cent. of extract of hemlock bark, 30 per cent of white precipitate and $\mathbf{1 0}$ per cent. of plaster-mass. By virtue of the alum and ergot which it contains, this preparation is astringent while the hemlock and white precipitate render it an excellent application when it is desirable to stimulate the absorbent. vessels. This preparation checks the discharge and allays the pain of open cancer. It promotes the absorption of lupus nodules, and encourages the cicatrization of lupus ulcers. It is a serviceable plaster in scrofulous patches, enlarged glands and scrofuloderma. Its astringent properties render it valuable in hyperidrosis and bromidrosis.

Aristol.-Aristol, 25 to 40 per cent., plaster mass, 75 to 60 per cent. Aristol plaster is reliable in the treatment of scrofuloderma, lupus, tuberculosis of the skin, ulcers, chronic eczema, psoriasis, acne, rosacea and syphilitic ulcers.

Belladonna and boric acid.-30 per cent. of belladonna and 20 per cent. of boric acid are mixed with 50 per cent. of plaster-mass. The action of the belladonna is assisted by the boric acid of this preparation. It is especially appropriate to painful affections. I have used it with decided satisfaction in recurrent herpes, herpes zoster, neuralgia and hyperæsthesia of the skin. It is of service, likewise, in cutaneous anæsthesia. Local spots of pruritis are relieved by the application of this plaster. It is of utility, also, in isolated spots of erythema, of subacute and chronic eczema, in which it lessens both the itching and infiltration. This is a good plaster for use in cases of local sweating, such as excessive and fetid perspiration of hands and feet, leading to maceration of the epithelium, and a fissured and painful condition of the integument. In fissured eczema, which especially attacks the fingers and toes, bella. donna and boric acid plaster is of noless value. To limited and chronic patches of vegetable parasitic disease, especially ringworm and favus, I have found

* These medicinal plasters named are prepared by Robert Young chemist, 1600 Chestuut street, Philadelphis, $\mathrm{Pa}$ 
this plaster a decidedly useful dressing. The boric acid destroys the organism while the belladonna assuages irritation. In cases of inflammation of the mammary gland belladonna is particularly useful and its effect in arresting the disease and checking secretion is enhanced by the combination with boric acid. This plaster is also useful in the treatment of bolls, abscesses, carbuncles, inflamed glands, burns, scalds, frost-bites, wounds and ulcers.

Cade.-Oil of cade, 25 per cent., beeswax, 25 per cent., plaster mass, 50 per cent. I have often used cade ointment with success in limited spots of chronic acne, rosacea, eczema, psoriasis and in old ulcers.

Camphor and opium.--Powdered camphor, 10 per cent., powdered opium, 5 per cent., olive oil., 5 per cent., plaster mass, 80 per cent. This is a useful combination, especially in painful affections, such as herpes, herpes zoster, and boils. It is also beneficial in burns, frost-bites, dermatitis, fissured nipples and bed-sores.

Chrysarobin.-Chrysarobin plaster contains 35 per cent of chrysarobin in 65 per cent. of plaster-mass. It is slightly stimulant and astringent and promotes absorption. The chrysarobin is capable in a higher proportion of causing painful erythema but in the strength here cited I have not witnessed irritation from its employment. Crysarobin plaster is particularly adapted to the treatment of rebellious affections, such as psoriasis, chronic eczema, chronic acne and rosacea. This plaster is preferably kept in place for several days though it is beneficial even when it can only be applied during the night. It is serviceable in the case of old scrofulous and syphilitic spots upon the skin. Chrysarobin plaster is efficacious in the different forms of ringworm.

Elm.-Resin elm, strained, 50 per cent., plaster mass, 50 per cent. Elm plaster is serviceably applied to wounds, ulcers, bed-sores, syphilitic and scrofulous spots, acne, rosacea, freckles and chloasma.

Hydrastin.-A plaster made of 30 per cent. of hydrastin and 70 per cent. of plaster-mass, is moderately stimulant and astringent and is useful in the treatment of chancroid, unhealthy and sloughing sores, cancers and excessive secretion of the skin.

Iodoform.-This preparation contains 40 per cent. of iodoform to 60 per cent of plaster-mass. Aristol, europhen or iodol may often be satisfactorily substituted for iodoform. Iodoform plaster possesses anæsthetic and antiseptic virtues. It is an admirable application to chancroids, ulcerated chancres, scrofulous and syphilitic spots and ulcers, bed-sores and wounds. This preparation may be used to arrest beginuing inflammation, as in boils, abscesses, adenitis and carbuncle and is beneficial in chronic ezema and lupus vulgaris.

Iodol.-Iodol, 40 per cent., plaster mass, 60 per cent. Iodol plaster is efficient in the treatment of syphilitic, scrofulous and tuberculous patches, chloasma, lentigo, scars and lupus.

Lead.-Lead plaster consists of 30 per cent. of oxide of lead and 70 per cent. of plaster-mass. It is one of the oldest and most useful preparations of this class. As a dressing in chronic eczema it serves an excellent purpose and is especially useful in cases attended by considerable thickening and a fissured condition of the skin. Lead plaster is often a good application, also, in subacute ezema. Erythema, burns, frost-bites and impetigo are among the affections relieved by the use of lead plaster.

Lead and opium.-Oxide of lead, 30 per cent., extract of opium, 10 per cent. and plaster-mass, 60 per cent., are contained in the above preparation. This plaster possesses the properties due to the lead and is applicable to the same cases as the simple lead plaster while the addition of the opium renders it anodyne. It is, therefore, peculiarly appropriate to the treatment of painful inflammatory conditions, such as boils, carbuncles, abscesses, bed-sores and irritable ulcers. Lead and opium plaster may be used with good effect, also, in herpes zoster, urticaria and paræsthesia.

Mercurial.-Mercurial plaster is composed of 50 per cent. each of red oxide of mercury and plaster-mass. It has a wide range of applicability. It is germicidal and sorbifacient, a cleanly and elegant application, and superior to mercurial ointment. It is beneficially applied to old syphilitic patches, to ulcers and scrofulous formations. Mercurial plaster diminishes pigmentation, and is therefore serviceable in freckles, and particularly to patches of chloasma. It is employed with benefit in lupus, acne and elephantiasis. Scars and hard infiltrated spots which result from inflammatory affections, as boils, carbuncles, sycosis and small-pox, are softened by the influence of mercurial plaster. In the treatment of many affections mercurial plaster is improved by the addition of 20 per cent. of extract of belladonna and 10 per cent. each of boric acid and extract of opium. Mercurial plaster may be beneficially applied to warts, burns, corns, callosities, chronic eczema and psoriasis. It is also used to promote absorption in enlargement of glands and joints.

M. Quinquaud has recently utilized a mercurial ointment in the treatment of constitutional syphilis. The formula of his preparation is as follows: Calomel, 20 ounces, castor oil, 6 ounces; and diachylon plaster, 60 ounces. The materials are thoroughly mixed and made into plasters four inches square. The directions given for use are that the skin is first to be washed with soap and water, after which a plaster is applied and allowed to remain upon the surface for eight days. Mercury begins to appear in the urine four or five days after the plaster is put on, and gradually increases, reaching its maximum on the eighth to eleventh day. The metal can be detected in the urine for the period of a month or six weeks. After the lapse of eight days M. Quinquaud is accustomed to have it discontinued and the skin left free for the space of eight days. This alternate use and disuse of the plaster in periods of eight. days is said to entirely avoid the occurrence of ptyalism.

Oxide of zinc.-This plaster contains 40 per cent. of oxide of zinc in 60 per cent. of plaster-mass. Oxide of zinc plaster is a most excellent preparation for use in subacute and chronic eczema. It is especially serviceable in fissured conditions. Strips of this plaster are extremely serviceable in fissures of the lip caused by eczema. This plaster can be recommended in the treatment of acne spots, seborrhœa, herpes, persistent boils and obstinate ulcers.

Oxide of zinc and camphor.-Camphor, 5 per cent., oxide of zinc, 40 per cent., plaster mass, 55 per cent. The foregoing combination relieves pain and itching. It is useful in chronic eczema, especially when itching is a prominent symptom. This preparation also 
answers a good purpose in erythema, and in boils and carbuncles attended with a high degree of inflammation.

Oxide of zinc and white precipitate-Oxide of zinc, 30 per cent., white precipitate, 15 per cent., plaster mass, 55 per cent. The presence of ammoniated mercury heightens the astringency of the oxide of zine and renders this preparation sorbifacient. It may be used with good results in cases of irritable scrofulous or syphilitic patches. It is likewise useful in chronic eczema.

Phytolacca and belladonna.-Extract of phytolacca, 20 per cent., extract of belladonna, 20 per cent., plaster-mass, 60 per cent. A plaster consisting of phytolacca and belladonna has a marked effect in reducing inflammation. It likewise promotes the repair of ulcers. Dermatitis, burns, ulcers, infiltrated and chronic patches of eczema, are relieved by the application of this plaster. The presence of phytolacca and belladonua renders it of particular value as a local remedy in mastitis. This preparation will often abort boils and inflammation of lymphatic glands if applied in the incipiency of those affections.

Subiodide of bismuth.-A plaster containing 30 per cent. of the subiodide of bismuth to 70 per cent. of plaster mass is useful. Subiodide of bismuth is not irritant, it has some local anæs thetic power, is astringent and promotes absorption. It likewise possesses antiseptic virtues. This plaster may be beneficially placed upon scrofulous or syphilitic spots. It has a good effect in freckles, chloasma and rosacea. It stimulates the absorption of inflammatory deposits, as indurated patches remaining after inflammation of the skin, orchitis, bubo and glandular enlargements.

Salicylic acid.-A plaster is made containing 25 per cent. of salicylic acid in 75 per cent. of plaster mass. It is germicidal and sorbifacient. I have found it excellently adapted to the treatment of hyperidrosis, bromidrosis, eczema of the palms and soles with thickening of the skin. It removes infiltration caused by psoriasis, boils and carbuncles. Salicylic acid plaster is a useful application in erysipelas and scrofuloderma. Dr. Hermann G. Klotz, of New York, writes that he has used with much satisfaction a plaster composed of salicylic acid, 5 to 10 per cent., petrolatum, 15 per cent., and 40 per cent. each of simple diachylon plaster and soap plaster. This, by suppressing evaporation of secretions, prevents the formation of crusts, cracking of the skin, and is useful in the treatment of rhagades. Dr. Klotz has found his compound salicylic acid plaster useful in recent vesicular and pustular eczema, and also in moist eczema of some duration, in old, infiltrated eczema, in chronic leg ulcers, syphilitic ulcers, pemphigus, lupus vulgaris and erythematosus.

Salicylic acid and cannabis indica.-25 per cent. of salicylic acid, 20 per cent. of extract of cannabis indica, and 55 per cent. of plaster mass, forms a combination which I have employed with excellent results in the treatment of corns, callosities, horns, warts, acne, rosacea, chronic eczema and psoriasis.

Salicylic acid and creosote.-Salicylic acid, 25 per cent., creosote, 5 per cent., plaster mass, 70 per cent. This is a good combination. The creasote mitigates the irritant and stimulant effect of the acid. A plaster thus composed is useful in lupus, chronic ulcers, hyperidrosis and bromidrosis.

Sulphur and chamomile.-Sulphur, 30 per cent., chamomile, 10 per cent., plaster mass, 60 per cent. In the foregoing combination the properties of both drugs are combined. Sulphur and chamomile plaster is a serviceable application in seborrhœa oleosa, papular eczema, acne and functional disorders of the sudoriparous glands.

Zinc chloride.-Chloride of zinc, 25 per cent., glycerin, 5 per cent., plaster mass, 70 per cent. This plaster is valuable in epithelioma, lupus vulgaris, and in old syphilitic and scrofulous ulcers.

In addition to the preceding formuix, many other drugs are usefully applied in the form of plasters. Aconite, aconite and belladonna, Peruvian balsam, and soap plasters are beneficial in frost-bites. A copper plaster is efficient in local spots of syphilis, scrofula, keloid, warts, burns, corns and callosities. Witch hazel is advantageous in chronic eczema and psoriasis. Arnica, asafotida or iron may be used in plaster form for the cure of indolent ulcers and bedsores. A plaster containing opium and iodine frequently aids removal of deep infiltratıons, especially in syphilitic subjects.

Unna has introduced into dermatological practice a class of preparations which he denominates plastermulls. They are recommended in cases where the Cisease is deeply seated and the atrongest applications are necessary. A layer of the medicament is evenly spread upon a sheet of gutta-percha tissue which is incorporated with a sheet of mull. In order to cause the drug to adhere both to the plaster and the skin, Unna makes use of the purest India rubber and purified oleate of aluminium. Both these substances are indifferent and unchangeable. By this method drug, plaster and skin are closely bound together. The drugs generally incorporated are mercury, carbolic acid, resorcin, salicylic acid and creosote, and oxide of zinc.

\section{A CASE OF LUPUS.}

Read in the Section of Dermatology and Syphilography, at the Fortythird annual meeting of the American Medical Association,

$$
\text { BY W. F. BREAKEY, M.U., }
$$

One of the purposes of this paper is to illustrate the need of delaying the report of a case until sufficient time has elapsed to have a fair and complete report.

The following memoranda of a case of lupus vulgaris, treated by Koch's lymph, May and June, 1891, with a postscript, April, 1892, are reported from notes made a year ago, as a contribution to the use of tuberculin, without comments or commendation, believing the facts to be of sufficient interest to warrant publication. Had it been reported a year ago a much more sanguine color might have been given. The inquiry suggests itself, how many of the published cures made a year ago would hold out now.

It should be stated that a case in the University Hospital, diagnosed as lupus, though not typical, had been treated for a short time with the lymph, but with unsatisfactory results, and another outpatient, with quite a typical case, was unwilling to try the treatment. I was, therefore, the more desirous to test the method in this patient, who was not only willing, but anxious to have the lymph tried. 